\title{
Judicialização do acesso a medicamentos no município de Ivinhema, Mato Grosso do Sul
}

Judicialization of access to medicines in the municipality of Ivinhema, Mato Grosso do Sul, Brazil

Judicialización del acceso a medicamentos en la ciudad de Ivinhema, Mato Grosso do Sul, Brasil

Tiago Branco Dias ${ }^{1}$

Fabiana Nabarro Ferraz ${ }^{2}$

Sheila Cristina Rocha Brischiliari ${ }^{3}$

Marco Antônio Costa ${ }^{4}$

\section{Resumo}

Objetivo: conhecer o perfil das requisições de medicamentos na esfera administrativa, recebidas pela Secretaria de Saúde do Município de Ivinhema, Mato Grosso do Sul, no ano de 2017. Métodos: estudo descritivo, retrospectivo, com foco em base documental. Definiuse como universo da pesquisa os ofícios recebidos pelo departamento jurídico da Secretaria de Saúde no Município de Ivinhema/MS, no período de primeiro de janeiro a 31 de dezembro de 2017. As variáveis concatenadas foram: quantificação das requisições de medicamentos no período supracitado; classificação da autoria dos ofícios recebidos; características dos medicamentos requeridos: pertinência à rede de assistência farmacêutica do SUS e classificação pela Anatomical Therapeutic Chemical Classification (ATC), de acordo com a recomendação da Organização Mundial de Saúde. Resultados: foram recebidos 59 ofícios, requisitando um total de 169 medicamentos. Desse total, $118(69,8 \%)$ não faziam parte da rede de assistência farmacêutica do SUS. Dos $51(30,2 \%)$ medicamentos listados na rede pública de saúde, 38 (74,5\%) estavam elencados na Relação Estadual de Medicamentos Essenciais (Resme) e 13 (25,5\%), na lista do componente especializado. Os fármacos solicitados com maior frequência foram: ácido acetilsalićlico (3,5\%), metoprolol e cilostazol $(2,4 \%)$. A maioria das requisições recebidas veio da Defensoria Pública $(93,2 \%)$. Além disso, segundo a classificação terapêutica dos medicamentos solicitados, verificou-se que os grupos anatômicos mais frequentes foram: sistema nervoso central $(31,9 \%)$, sistema cardiovascular (26,0\%) e sangue e órgãos hematopoiéticos (10,6\%). Conclusão: a maioria dos ofícios era de autoria da Defensoria Pública e requisitavam medicamentos que não faziam parte da rede de assistência farmacêutica do SUS.

Palavras-chave: Direito à saúde. Medicamentos. Judicialização da saúde.

\footnotetext{
${ }^{1}$ Especialista em Gestão em Saúde; farmacêutico da Coordenação da Assistência Farmacêutica, Ivinhema, Mato Grosso do Sul, Brasil. E-mail: tbdias@hotmail.com

2 Doutora em Ciências da Saúde; farmacêutica-bioquímica no Departamento de Análises Clínicas e Biomedicina da Universidade Estadual de Maringá, Maringá, Paraná, Brasil. E-mail: fabiana_nabarro@hotmail.com

${ }^{3}$ Doutora em Ciências da Saúde; professora de Enfermagem na Universidade Estadual do Oeste do Paraná, Foz do Iguaçu, Paraná, Brasil. E-mail: sheila.brischiliari@gmail.com

${ }^{4}$ Doutor em Ciências Farmacêuticas; professor do Departamento de Farmácia da Universidade Estadual de Maringá, Maringá, Paraná, Brasil. E-mail: macosta@uem.br
} 


\begin{abstract}
Objective: to know the profile of medicines requests in the administrative level, received by the Secretary of Health of Ivinhema, Mato Grosso do Sul, Brazil, in 2017. Methodology: descriptive, retrospective study, focusing on documentary basis. The universe of research was defined as the documents received by the law department of Secretary of Health of Ivinhema, Mato Grosso do Sul, from January 1 to December 31, 2017. The variables studied were: quantification of medicines requests; authorship classification of the received documents; characteristics of the required medicines: relevance to the SUS pharmaceutical assistance network and classification by the Anatomical Therapeutic Chemical Classification (ATC), according to the recommendation of the World Health Organization. Results: 59 documents were received, requesting a total of 169 medicines. Of this total, $118(69.8 \%)$ were not part of SUS's pharmaceutical assistance network. Of the 51 (30.2\%) medicines listed in the public health network, 38 (74.5\%) were listed in the State List of Essential Medicines (Resme), and $13(25.5 \%)$ in the specialized component list. The most frequently requested medicines were acetylsalicylic acid (3.5\%), metoprolol and cilostazol (2.4\%).Most of the documents received came from the Public Defender's Office (93.2\%). In addition, according to the therapeutic classification of the medicine requested, it was found that the most frequent anatomical groups were: central nervous system (31.9\%), cardiovascular system (26.0\%) and blood and hematopoietic organs (10.6\%). Conclusion: Most of the documents were from the Public Defender's and requested medicines that were not part of SUS's pharmaceutical assistance network.
\end{abstract}

Keywords: Right to health. Medicines. Health's. Judicialization.

\title{
Resumén
}

Objetivo: conocer el perfil de las requisiciones de medicamentos en la esfera administrativa, recibidas por la Secretaría de Salud del Municipio de Ivinhema, Mato Grosso do Sul, en el año 2017. Metodología: estudio descriptivo, retrospectivo con foco en base documental. Se definió como universo de la investigación los oficios recibidos por el departamento jurídico de la Secretaría de Salud en el Municipio de Ivinhema/MS, en el período del primero de enero al 31 de diciembre de 2017. Las variables concatenadas fueron: cuantificación de las solicitudes de drogas en el período mencionado; clasificación de autoría de los oficios recibidas; características de los medicamentos requeridos: relevancia para la red de atención farmacéutica del SUS y clasificación según la Clasificación Química Terapéutica Anatómica (ATC), según la recomendación de la Organización Mundial de la Salud. Resultados: recibieron 59 oficios, solicitando un total de 169 medicamentos. De este total, $118(69,8 \%)$ no formaban parte de La red de Asistencia Farmacéutica del SUS. De los 51 (30,2\%) medicamentos listados en la red pública de salud, 38 (74,5\%) estaban enumerados en la Lista Estatal de Medicamentos Esenciales (Resme) y 13 (25,5\%) en la lista del componente especializado. Los fármacos solicitados com mayor frecuencia fueron: ácido acetilsalicílico $(3,5 \%)$, metoprolol y cilostazol (2,4\%). La mayoría de las acciones recibidas vinieron de La Defensoría Pública (93,2\%). Además, según La clasificación terapéutica de los medicamentos solicitados, se verificó que los grupos anatómicos más frecuentes fueron: sistema nervioso central (31,9\%), sistema cardiovascular $(26,0 \%)$ y sangre y órganos hematopoyéticos (10,6\%). Conclusion: La mayoría de los documentos provenían de la Oficina del Defensor Público y solicitaban medicamentos que no formaban parte de la red de asistencia farmacéutica del SUS. 
Palabras-llave: Derecho a la salud. Medicamentos. Judicialización de La salud.

\section{Introdução}

A política de assistência farmacêutica do Sistema Único de Saúde (SUS) é definida por diretrizes de gestão que visam a assegurar o provimento e o uso coerente de medicamentos (1).

No Brasil, o acesso ao medicamento é direito do cidadão, pois se trata do direito à saúde, sendo ele um bem importante para o cuidado, pelo seu potencial para prevenir e curar doenças, bem como salvar vidas (2).

A garantia do direito à saúde deve se dar por meio de políticas sociais e econômicas, não por meio de decisões judiciais. Entretanto, o processo de busca judicial por esse direito se iniciou nos anos 90, a partir de solicitação de fármacos antiretrovirais para o tratamento do HIV/Aids, onde os medicamentos eram caros e de difícil acesso. Essa demanda, unida à recente aprovação da Constituição da República Federativa do Brasil (CRFB) em 1988, tornou muito frequente a reclamação judicial por bens e serviços de saúde. A essa nova e constante atuação do Judiciário na garantia de direitos individuais tem sido atrelada a noção de judicialização (3).

Judicialização significa que as questões que deveriam ser decididas pelos gestores em saúde estão sendo decididas pelo Poder Judiciário. Porém, também pode ser vista como o único caminho para combater a omissão dos gestores e sistemas de saúde implantados. A judicialização da saúde envolve vários setores, criando um sistema conjunto de operações entre os sistemas de justiça e de saúde $(4,5)$.

Verifica-se, no Brasil, um aumento do número de decisões judiciais, que submete ao Poder Público o fornecimento de bens e serviços de saúde. Isso é facilmente comprovado quando se analisa os dados do Conselho Nacional de Justiça (CNJ). Houve um crescimento superior a 350\% quando se compara o número de processos em 2011 (240.980) em relação ao ano de 2016 (854.506) (4). Um dos principais produtos de saúde reivindicados nos tribunais são os medicamentos. As crescentes demandas judiciais contra entidades públicas no país associadas aos fármacos demonstram que o Poder Judiciário vem se consolidando como um meio para que o cidadão garanta o seu direito à saúde (6).

Sabendo da importância desse assunto na administração em saúde, no que diz 
respeito às políticas públicas, o presente estudo tem por objetivo conhecer o perfil das requisições de medicamentos na esfera administrativa, recebidas pela Secretaria de Saúde do Município de Ivinhema, Mato Grosso do Sul, no ano de 2017.

\section{Métodos}

Trata-se de um estudo descritivo, retrospectivo, com foco em base documental. Definiu-se como universo da pesquisa as requisições de medicamentos na esfera administrativa, recebidas pelo departamento jurídico da Secretaria de Saúde no Município de Ivinhema/MS, no período de primeiro de janeiro a 31 de dezembro de 2017. Por esfera administrativa, entende-se o recebimento dos ofícios gerados por órgãos competentes com os pleitos alusivos aos medicamentos, situação que antecede uma possível ação judicial.

A tramitação se inicia quando o departamento jurídico da Secretaria de Saúde, no Município de Ivinhema/MS, recebe o ofício da Defensoria Pública do Estado com a requisição de medicamentos. O documento é então encaminhado ao farmacêutico coordenador da Assistência Farmacêutica para que seja emitido um relatório especificando informações sobre os fármacos solicitados, como, por exemplo, a pertinência em listas do SUS. Portando o relatório farmacêutico, o departamento jurídico realiza a devolutiva com suas alegações, ficando a instrução de ação judicial a critério da Defensoria Pública.

O envio desses ofícios por parte da Defensoria Pública, instituição essencial à função juridisdicional, foram considerados, para este estudo, como o princípio do processo de judicialização.

Segundo o último censo do IBGE (2010), Ivinhema tem 22.341 habitantes. Sua estrutura relacionada à saúde pública é composta por um hospital, um centro de especialidades médicas, uma unidade básica de saúde e oito estratégias saúde da família (ESF).

As seguintes variáveis foram concatenadas para o estudo: quantificação das requisições de medicamentos no período supracitado; classificação da autoria das ações recebidas; e características dos medicamentos requeridos, como pertinência à rede de assistência farmacêutica do SUS e classificação pela Anatomical Therapeutic Chemical Classification (ATC) $)^{5}$, de acordo com a recomendação da Organização Mundial de Saúde

\footnotetext{
${ }^{5}$ Disponível em https://www.whocc.no/atc_ddd_index/
} 
(OMS).

Nas situações em que o medicamento solicitado estava descrito pelo nome comercial, o nome do fármaco (ou associação medicamentosa) foi obtido por meio do site Kairos ${ }^{2}$.

Para fins da seleção oficial do elenco de medicamentos contidos na regulamentação da assistência farmacêutica no SUS, considerou-se o elenco da Relação Estadual de Medicamentos Essenciais (Resme) de 2017, vigente no estado do Mato Grosso do Sul na ocasião do estudo, assim como a Lista do Componente Especializado, regida pela Portaria de consolidação no 1/2017 (7).

\section{Resultados e Discussão}

No período estudado, foram recebidos 59 ofícios (média de 4,9 ofícios/mês), requisitando um total de 169 medicamentos (média de 2,9 medicamentos/solicitação). Desse total, $118(69,8 \%)$ não faziam parte da rede de assistência farmacêutica do SUS (Tabela 1). Alguns princípios ativos, apesar de constarem na relação pública, destoavam quando se analisava a concentração e/ou forma farmacêutica. Entre os itens solicitados não padronizados, destacou-se a maior frequência de fármacos relacionados ao sistema nervoso central $(42 / 118,35,6 \%)$ e sangue e órgãos hematopoiéticos (13/118, 11,0\%), segundo a classificação do primeiro nível da Anatomical Therapeutic Chemical Classification (ATC).

Tabela 1. Itens de medicamentos requisitados via ofício em relação à presença ou não na Rede de Assistência Farmacêutica do SUS, município de Ivinhema, Mato Grosso do Sul, 2017

\begin{tabular}{ll}
\hline Medicamentos Solicitados & Frequência total dos itens \\
\hline $\begin{array}{ll}\text { Itens de medicamentos que pertencem à Rede de } \\
\text { Assistência Farmacêutica do SUS }\end{array}$ & $51(30,2 \%)$ \\
$\begin{array}{l}\text { Itens de medicamentos que não pertencem à Rede } \\
\text { de Assistência Farmacêutica do SUS }\end{array}$ & $118(69,8 \%)$ \\
\hline Total & $169(100 \%)$ \\
\hline
\end{tabular}

Fonte: Elaboração própria com base nos dados da pesquisa.

Dos 51 (30,2\%) medicamentos listados que estão previstos na rede pública de saúde,

\footnotetext{
${ }^{2}$ Disponível em http://brasil.kairosweb.com/
} 
38 (74,5\%) faziam parte do componente básico, ou seja, de competência do município e estavam elencados na lista padronizada para fornecimento, a Resme do estado do Mato Grosso do Sul e 13 (25,5\%) na lista do componente especializado. Essa linha de cuidado engloba fármacos geralmente adquiridos pelo estado, que detém ainda a responsabilidade de armazenamento e distribuição para os municípios.

Nos estados da Bahia (6), Rio de Janeiro (8) e São Paulo (9), estudos demonstraram um grande contingente de ações judiciais solicitando medicamentos que fazem parte das listas oficiais de distribuição pública no SUS. Conforme analisado, a demanda também foi superior a $30 \%$.

O percentual de medicamentos solicitados pela Defensoria Pública, e que pertencem às listas oficiais do SUS (29,6\%), remete às seguintes hipóteses: i) o indivíduo não obteve o fornecimento ao recorrer ao órgão de saúde; ii) não buscou o recebimento ou evocou o acesso dos medicamentos de seu receituário de forma integral, no qual poderiam estar prescritos fármacos padronizados; iii) desconhecimento do prescritor sobre a disponibilidade dos medicamentos que fazem parte das listas oficiais de distribuição pública no SUS. Hipóteses semelhantes foram levantados em estudos realizados por Oliveira et al (6) e Borges e Ugá (8).

Na Secretaria de Saúde do Município de Ivinhema/MS, a aquisição de medicamentos é realizada por intermédio do departamento de compras, que conta com uma equipe técnica devidamente treinada para o exercício das atividades. O processo licitatório é realizado no mês de dezembro, para contratualização no ano seguinte. Todavia, o não recebimento do componente básico pode ser explicado pelo eventual desabastecimento, em virtude da aquisição, que pode esbarrar em imprevistos inerentes ao processo, tais como: indisponibilidade de estoque regulatório da empresa licitada; solicitação de troca de marca; item fracassado ou deserto na licitação; e indisponibilidade de matéria-prima no mercado.

Já no especializado, é comum o relato de pacientes que apresentam uma condição clínica que não está associado a uma classificação internacional de doenças (CID) da lista do componente especializado, regida pela Portaria de Consolidação no 1/2017 do Ministério da Saúde (7). Nesses casos, os medicamentos só serão fornecidos aos pacientes via determinação judicial.

Em relação à autoria, das 59 requisições, 55 (93,2\%) vieram da Defensoria Pública, 
2 (3,4\%) do Ministério Público e 2 (3,4\%) do Conselho Tutelar (Figura 1). Estudos realizados no estado do Rio de Janeiro (10,11), Bahia (6), Distrito Federal (12) e Pará (13) também evidenciaram o predomínio de ações patrocinadas pela Defensoria Pública.

Figura 1. Autoria dos ofícios com requerimento de medicamentos do município de Ivinhema, Mato Grosso do Sul, 2017

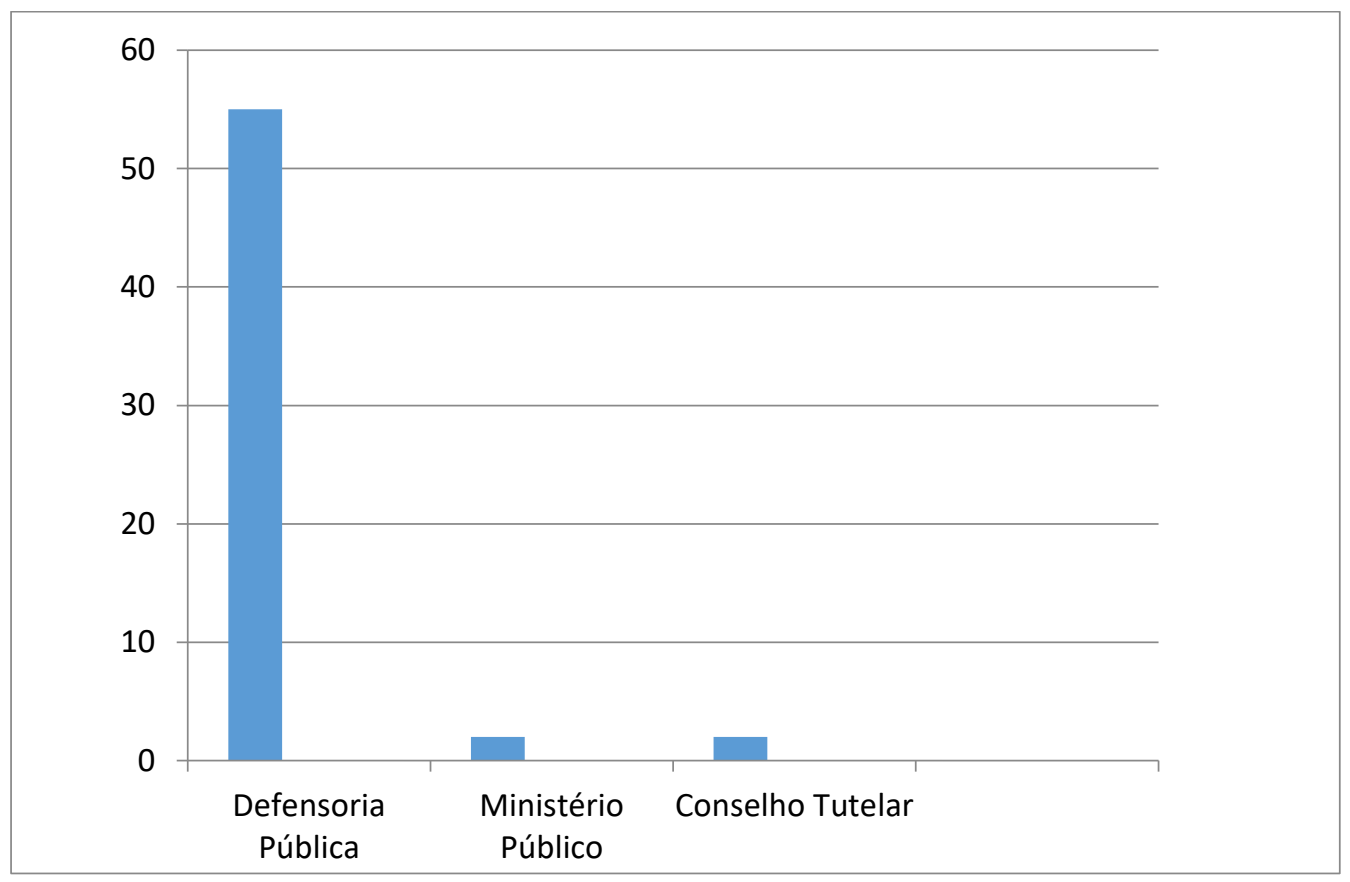

Fonte: Elaboração própria com base nos dados da pesquisa.

O grande porcentual de ofícios de autoria da Defensoria Pública é explicado, provavelmente, pela facilidade de acesso. O município estudado dispõe da $1^{\underline{a}}$ e $2^{\underline{a}}$ Defensoria Pública Estadual. Ambas prestam serviços efetivos à população, no qual se inclui a área da saúde, atuando também de forma extrajudicial. Existe a possibilidade de concessão do benefício da justiça gratuita ao usuário, oferecida por esse órgão desde que os usuários sejam economicamente hipossuficientes e que efetivamente comprovarem essa situação (14).

Com relação à classificação terapêutica dos medicamentos solicitados, segundo as normas da Anatomical Therapeutic Chemical Classification (ATC), verificou-se que os grupos anatômicos mais frequentes foram: sistema nervoso central $(31,9 \%)$, sistema cardiovascular (26,0\%), sangue e órgãos hematopoiéticos (10,6\%) (Tabela 2). 
Estudo realizado em 2010 - ao classificar os grupos anatômicos pelo primeiro nível, de acordo com a ATC - apresentou um resultado similar ao obtido neste estudo, pois somados os medicamentos dos sistemas cardiovascular e nervoso, resultaram mais de $50 \%$ das solicitações (11).

Tabela 2. Distribuição dos medicamentos solicitados segundo classificação pelo primeiro nível do Anatomical Therapeutic Chemical (ATC), no município de Ivinhema, Mato Grosso do Sul, em 2017

\begin{tabular}{lcc}
\hline Primeiro nível da ATC & № & $\%$ \\
\hline Sistema nervoso central & 54 & 31,9 \\
Sistema cardiovascular & 44 & 26,0 \\
Sangue e órgãos hematopoiéticos & 18 & 10,6 \\
Aparelho digestivo e metabolismo & 14 & 8,3 \\
Sistema músculo esquelético & 11 & 6,5 \\
Sistema Respiratório & 7 & 4,1 \\
Anti-infecciosos gerais para uso sistêmico & 6 & 3,5 \\
Dermatológico & 5 & 3,0 \\
Órgãos dos sentidos & 4 & 2,4 \\
Antineoplásicos e agentes moduladores do sistema & 3 & 1,8 \\
imunológico & 2 & 1,2 \\
Hormônios de uso sistêmico, excluindo hormônios sexuais & 1 & 0,6 \\
Não classificável & 169 & 100 \\
\hline Total & & \\
\hline
\end{tabular}

Fonte: Elaboração própria com base nos dados da pesquisa.

O efetivo acesso dos pacientes aos medicamentos da rede pública esbarra em uma série de fatores, dos quais se destacam a não adesão ou o desconhecimento da lista, por parte dos prescritores; o desabastecimento temporário; e a avidez de venda da indústria farmacêutica desses ativos não padronizados (15).

Quando o setor público dispensa um medicamento não contemplado por uma lista oficial, abre-se o precedente da destinação de recursos públicos em benefício individual em detrimento do coletivo. Insta salientar que as padronizações e os protocolos clínicos refletem 
eficácia e segurança ao paciente, haja vista que são fundamentados em evidências clínicas comprovadas e disponíveis na literatura científica (16).

Em seção realizada em abril de 2018, o Supremo Tribuna Federal (STF) definiu três requisitos cumulativos para a concessão de fármacos não inseridos nas regulamentações do SUS: laudo do médico que assiste o paciente que fundamente e detalhe a indispensabilidade ou necessidade do medicamento, bem como a infrutuosidade dos fármacos do SUS para o tratamento da enfermidade; e inexistência de condição financeira para arcar com o medicamento prescrito e que este tenha registro na Agência Nacional de Vigilância Sanitária (Anvisa). Decidiu ainda que, após o trânsito em julgado de cada processo, sejam comunicados tanto o Ministério da Saúde, como a Comissão Nacional de Tecnologias do SUS (Conitec), no sentido que sejam realizados estudos acerca da viabilização desses medicamentos reivindicados na esfera do SUS (17).

Em decisão recente, o STF desobrigou o Estado de fornecer medicamentos que não apresentem registro na Anvisa, ou seja, que estejam em fase de experimentos. Somente quando a Anvisa for a responsável pelo não registro, como levar muito tempo para apreciar uma solicitação, o medicamento poderá ser fornecido, obedecendo três condições: já existir solicitação de registro do medicamento no Brasil (exceto para doenças raras); 0 medicamento já ser registrado no exterior; e a inexistência de medicamento substituto (18).

Como essa decisão tem repercussão geral, muitos casos judicializados hoje deixarão de ser atendidos e novos casos de medicamentos já registrados ganharão maior agilidade na decisão. Esse último fato exigirá dos gestores ações preventivas como a aquisição dos medicamentos mais solicitados, evitando e/ou diminuindo a judicialização no futuro próximo (18).

Apesar do Poder Executivo falhar em algumas situações, como permitir o desabastecimento de alguns medicamentos na atenção básica em determinados períodos, possivelmente a tomada de decisões das expensas em saúde pelo Executivo poderia otimizar a utilização dos recursos públicos destinados à judicialização, uma vez que detém o know-how tanto das necessidades, como dos pecúlios disponíveis para o atendimento das mesmas (5). 


\section{Considerações finais}

Este estudo demonstrou que a maioria dos ofícios recebidos, na esfera administrativa, pelo departamento jurídico da Secretaria de Saúde do Município de Ivinhema, Mato Grosso do Sul, no ano de 2017, eram de autoria da Defensoria Pública e requisitavam medicamentos que não faziam parte da rede de assistência farmacêutica do SUS. Além disso, também foi requisitada uma parcela significativa de medicamentos listados na rede pública de saúde, fato provavelmente relacionado ao desabastecimento temporário do medicamento que decorre, geralmente, de situações inerentes ao processo de licitação.

Assim, a análise do perfil das requisições de medicamentos, na esfera administrativa, torna-se importante para levantar questionamentos quanto à organização dos serviços, avaliar as necessidades epidemiológicas da população, bem como nortear os gestores públicos em suas ações.

\section{Referências}

1. Brasil. Assistência Farmacêutica no SUS / Conselho Nacional de Secretários de Saúde. Brasília: 2011.

2. Wannmacher L. Importância dos Medicamentos Essenciais em Prescrição e Gestão Racionais. In: Brasil. Uso Racional de Medicamentos: temas selecionados. Ministério da Saúde. Brasília: 2010.

3. Chieffi AL, Barata RB. Judicialização da política pública de assistência farmacêutica e equidade. Cadernos de Saúde Pública. 2009, 25 (8): 1839-1849. Disponível em: http://www.scielo.br/scielo.php?script=sci_arttext\&pid=S0102-311X2009000800020

[Acesso em 12.out.2018]

4. Santos AO, Schulze CJ. A situação atual e o futuro da judicialização da política de assistência farmacêutica no Brasil - O papel da farmacoeconomia. Jornal de Assistência Farmacêutica e Farmacoeconomia. 2016,1 $\quad$ (3): 7-9. Disponível em http://www.jaff.org.br/jornal/Upload/anexo_revista/EDITORIAL\%202.pdf [Acesso em 12.out.2018]

5. Barroso LR. Da falta de efetividade à judicialização excessiva: direito à saúde, fornecimento gratuito de medicamentos e parâmetros para a atuação judicial. Doutrina. Jurisp. Mineira, Belo Horizonte, a. 60, $\mathrm{n}^{\circ}$ 188, p. 29-60, 2009. Disponível em https://bd.tjmg.jus.br/jspui/bitstream/tjmg/516/1/D3v1882009.pdf [Acesso em: 26.nov.2018]

6. Oliveira CRV, Pereira NSG, Rodrigues FF, Sampaio LOS, Perrone MLCCM, Oliveira CRV. Perfil das demandas judiciais por medicamentos na diretoria de assistência farmacêutica da 
Bahia no ano de 2015. Jornal de Assistência Farmacêutica e Farmacoeconomia. 2016, 1(Suppl.1): 40. Disponível em http://www.jaff.org.br/jornal/Upload/anexo_revista/ANAIS\% 20JAFF\%202016\%20CORRIGIDO.48.pdf [Acesso em 12.out.2018]

7. Brasil. Portaria de consolidação no 1 , de 28 de setembro de 2017. Consolidação das normas sobre os direitos e deveres dos usuários da saúde, a organização e o funcionamento do Sistema Único de Saúde. Brasília. Disponível em: http://portalsinan.saude.gov.br/images/ documentos/Legislacoes/Portaria_Consolidacao_1_28_SETEMBRO_2017.pdf [Acesso em: 29.out.2019]

8. Borges DCL, Ugá MAD. Conflitos e impasses da judicialização na obtenção de medicamentos: as decisões de 1ํㅡㄹ instância nas ações individuais contra o Estado do Rio de Janeiro, Brasil, em 2005. Cadernos de Saúde Pública. 2010, 26(1): 59-69. Disponível em http://www.scielo.br/pdf/csp/v26n1/07.pdf [Acesso em 06.fev.2019]

9. Ferraz LMA. Judicialização da saúde: acesso a medicamentos na cidade de Botucatu, Estado de São Paulo, Brasil [Dissertação]. São Paulo: pós-graduação em saúde coletiva da Universidade Estadual Paulista, 2018. Disponível em: https://repositorio.unesp.br/handle/ 11449/153481 [Acesso em: 29.out.2019]

10. Messeder AM, Osorio-de-Castro CGS, Luiza VL. Mandados judiciais como ferramenta para garantia do acesso a medicamentos no setor público: a experiência do Estado do Rio de Janeiro, Brasil. Cadernos de Saúde Pública. 2005, 21(2): 525-534. Disponível em http://www.scielo.br/pdf/csp/v21n2/19.pdf [Acesso em: 12.out.2018]

11. Pepe VLE, Ventura M, Sant'ana JMB, Figueiredo TA, Souza VR, Simas L, Osorio-deCastro CGS. Caracterização de demandas judiciais de fornecimento de medicamentos "essenciais" no Estado do Rio de Janeiro, Brasil. Cadernos de Saúde Pública. 2010, 26(3): 461-471. Disponível em http://www.scielo.br/scielo.php?script=sci_arttext\&pid=S0102311X2010000300004 [Acesso em: 12.out.2018]

12. Romero LC. Judicialização das políticas de assistência farmacêutica: o caso do Distrito Federal. Brasília: Consultoria Legislativa do Senado Federal. 2008. Disponível em http://www2.senado.leg.br/bdsf/item/id/96829 [Acesso em: 12.out.2018]

13. Damasceno TV, Ribeiro KCB. Judicialização da saúde nos municípios da região metropolitana de Belém-PA. Cadernos Ibero-Americanos de Direito Sanitário. 2019, 8(2): 100-115. Disponível em https://www.cadernos.prodisa.fiocruz.br/index.php/cadernos/article/ view/516 [Acesso em: 29.out.2019]

14. Soares AA, Vidal CGM. Direito à saúde, escassez e judicialização: uma tríade de paradoxos [Dissertação]. Paraíba: Departamento de Serviço Social da Universidade Federal da Paraíba, 2017. Disponível em: https://repositorio.ufpb.br/jspui/bitstream/123456789/ 3053/1/AAS_CCGMV30102017.pdf [Acesso em: 29.out.2019]. 
15. Catanheide ID, Lisboa ES, Souza LEPF. Características da judicialização do acesso a medicamentos no Brasil: uma revisão sistemática. Physis: Revista de Saúde Coletiva. 2016, 26 (4): 1335-1356. Disponível em http://www.scielo.br/pdf/physis/v26n4/1809-4481-physis26-04-01335.pdf [Acesso em:12.out.2018]

16. Tanaka OY. A judicialização da prescrição medicamentosa no SUS ou o desafio de garantir o direito constitucional de acesso à assistência farmacêutica. Revista De Direito Sanitário. 2008, 9(1): 139-143. Disponível em http://www.revistas.usp.br/rdisan/article/ view/13109/14912 [Acesso em: 12.out.2018]

17. Brasil. Supremo Tribunal Federal (STF). Recurso especial № 1.657 .156 - RJ (2017/0025629-7), Ementa - Fornecimento pelo poder público de medicamentos que não consta em atos normativos do SUS. 2018. Disponível em: https://ww2.stj.jus.br/processo/ revista/documento/mediado/?componente $=$ ITA\&sequencial $=1641175 \&$ num_registro $=20170$ 0256297\&data $=20180504 \&$ formato=PDF [Acesso em: 15. out.2019]

18. Lamarão Neto H. A regra de solidariedade dos entes federados na área da saúde e sua atual interpretação pelo Supremo Tribunal Federal. Cadernos Ibero-Americanos de Direito Sanitário. 2019, 8(3): 10-26. Disponível em: https://www.cadernos.prodisa.fiocruz.br/index.php/ cadernos/article/view/569/605 [Acesso em: 18.out.2019]

Como citar este artigo:

Dias TB, Ferraz FN, Rocha-Brischiliari SC, Costa MA. Judicialização do acesso à medicamentos no município de Ivinhema, Mato Grosso do Sul. Cadernos Ibero-Americanos de Direito Sanitário. 2019 out./dez.; 8(4): 66-77.

http://dx.doi.org/10.17566/ciads.v8i4.540 